\title{
§ 10 IVV: Vergütung der Geschäftsleiter
}

Der Entwurf der Auslegungshilfe zu dem unverändert gebliebenen § 10 IVV wurde um die Bestimmung ergänzt, dass in Bezug auf eine in Instrumenten zugewendete variable Vergütung angemessene Maßnahmen zu ergreifen sind, um die Unabhängigkeit des Urteils des Geschäftsleiters nicht zu beeinflussen. Konkret wird als Beispiel für eine solche Maßnahme die Festlegung von Verfügungssperrfristen bis zum Ablauf des Mandats genannt. Hier dürfte wohl nicht das Ende der jeweiligen turnusmäßigen Bestellung gemeint sein, sondern tatsächlich der Ablauf des Mandats, d. h. das endgültige Ausscheiden aus dem Amt als Vorstand oder Geschäftsführer.

Bedenken bereitet insoweit, dass Sperrfristen bei Risikoträgern in der Verordnung selbst geregelt sind, nämlich in $\S 20$ Abs. 5 S. 2 und in $\S 22$ Abs. 2 IVV. Damit hat der Verordnungsgeber deren Wichtigkeit zum Ausdruck gebracht, was sich nicht damit in Einklang bringen lässt, bei Geschäftsleitern nicht bedeutender Institute Sperrfristen in der Auslegungshilfe vorzusehen.

Es ist deshalb davon auszugehen, dass die finale Auslegungshilfe, sollte sie mit dem Entwurf der Auslegungshilfe insoweit identisch sein, die eine Außenwirkung eh nur über den allgemeinen Gleichbehandlungsanspruch im Zusammenschau mit der Rechtsfigur der Selbstbindung der Verwaltung entfaltet, insoweit rechtswidrig ist und damit streng genommen nicht befolgt $\mathrm{zu}$ werden braucht. Trotzdem werden jedenfalls von der BaFin beaufsichtigte Institute kaum die in der Auslegungshilfe formulierte Erwartung einer Sperrfrist ignorieren wollen. 